Brown-HET-1401

\title{
Moduli Stabilization with the String Higgs Effect
}

\author{
Scott Watson* \\ Physics Department, Brown University, Providence RI 02912 USA.
}

(Dated: September 5, 2018)

\begin{abstract}
We review the notion of the Higgs effect in the context of string theory. We find that by including this effect in time dependent backgrounds, one is led to a natural mechanism for stabilizing moduli at points of enhanced gauge symmetry. We consider this mechanism for the case of the radion (size of the extra dimensions) and find that as decompactification of the large spatial dimensions takes place the radion will remain stabilized at the self dual radius. We discuss how this mechanism can be incorporated into models of string cosmology and brane inflation to resolve some outstanding problems. We also address some issues regarding which string states should be included when constructing low energy actions in string cosmology.
\end{abstract}

PACS numbers:

*Electronic address: watson@het.brown.edu 


\section{INTRODUCTION}

Superstring theory predicts the existence of a number of massless scalar fields, known as moduli fields. These fields are troublesome for both string theory and string cosmology. From the string theory perspective they represent flat directions leading to a degeneracy in the vacuum state for the string, i.e. string theory fails to determine its own vacuum configuration. From the perspective of cosmology these massless scalars are problematic because they would have dramatic effects on the cosmological evolution, which seem to be in contradiction with observation. One resolution to the so-called cosmological moduli problem is to address these issues through considering models of string cosmology. In this way, one may argue that the real vacuum state is chosen among the many possibilities by considering the cosmological evolution.

One attempt at such a construction occurs in models of string gas cosmology, also known as brane gas cosmology (BGC) 1]. In these models, in addition to the massless modes of the string, one includes the massive modes in the form of a gas of string winding and momentum modes through their stress energy tensor. One can then show that the radion, the modulus giving the overall size of the extra dimensions, is fixed near the string scale by the cosmological evolution 2, 3]. Although these results are promising, it is somewhat inconsistent to include the massive modes into a low energy effective theory. Moreover, it was shown in [4] that at late times, when the dynamics are described by the $4 D$ effective theory, stabilization will only remain for the special case of one extra dimension. Both of these problems present serious challenges for models of BGC.

Another method for fixing moduli has been considered in flux compactifications of Type IIB string theory [5]. In these models it is possible to fix nearly all of the moduli by introducing fluxes wrapping the cycles of the Calabi-Yau. Moreover, it has been possible to construct slow roll inflation models in the warped geometry background where the role of the inflaton is played by the separation of the $D 3$ brane in the bulk and a stack of $\bar{D} 3$ branes at an IR fixed point [6]. One drawback of these models is the method for stabilizing 
the size of the extra dimensions seems to be incompatible with slow-roll inflation. It was suggested in [6] that this problem might be alleviated by considering additional methods for stabilizing the radion (size of the extra dimensions). The method we present here, motivates low energy corrections that should be added to the Kahler potential and results in an additional method for stabilizing the radion.

In this paper we consider the realization of the string Higgs effect in cosmology. We take as our starting point the massless modes of the string as described by the low energy effective action. We then find that there exists a critical radius at which there are additional massless modes. As one moves away from this point of enhanced gauge symmetry (ESP) one finds that these particles gain a mass in a manner analogous to that of electroweak symmetry breaking. By including the backreaction of these additional modes on the evolution, along with the damping of the cosmological expansion, we find a method for stabilizing the radion. This is similar to work recently discussed in [7], where in that paper the example was that of a two brane system and the ESP was related to the inter-brane separation.

In Section II, we discuss the necessary string theory background for constructing the cosmological model of interest. We review string compactifications from both the spacetime and string worldsheet conformal field theory (CFT) perspectives. We focus on the low energy spectrum and hope to convince the reader that one needs to be cautious when including string matter into low energy effective actions. In particular, we demonstrate that for a generic radius of compactification, no winding or momentum states should be present in the spectrum. We then consider the spectrum near an ESP, where the gauge theory of the string is enriched by additional massless states. This is an example of the Higgs effect in string theory [8] and when combined with a time dependent or cosmological background will result in interesting consequences. In particular, we demonstrate in Section III that by considering the additional massless states created at the ESP we can arrive at a natural mechanism for stabilizing the moduli of the theory. In this case the modulus of interest will be the radion, i.e. the radius of the extra dimensions, and by 
considering the production of massless string modes we find a natural way to dynamically determine the overall size of the extra dimensions. We will conclude with some brief remarks on how this work could be incorporated into current models of string cosmology.

\section{STRING COMPACTIFICATIONS}

In this section, we review the low energy effective action for the string, focusing on the bosonic or Neveu-Schwarz (NS) degrees of freedom. We want to focus in particular on which additional modes could in principle be added to the low energy action. We consider the additional modes that arise from compactification, or perhaps more appropriately from decompactification of the large $3+1$ space-time. We find that many of these modes become massless near so-called enhanced symmetry points (ESP). We will see that this results in a natural example of the Higgs effect in string theory [8] and including these modes back into the effective action results in non-trivial effects for the evolution of the radion.

We take as our starting point the low energy effective action for the NS-NS sector of the string in $D=4+d$ space-time dimensions (for a review see [11]),

$$
S_{D}=\frac{1}{2 \kappa_{0}^{2}} \int d^{4+d} x \sqrt{-G} e^{-2 \Phi}\left(R_{D}+4(\nabla \Phi)^{2}-\frac{1}{12} H^{2}+\mathcal{O}\left(\alpha^{\prime}\right)\right)+\mathcal{O}\left(g_{s}\right),
$$

where $H_{M N P}=\nabla_{[M} B_{N P]}$ is the three form flux, $\Phi$ is the dilaton, and $G_{M N}$ is the $D$

dimensional metric with $M, N=0 \ldots D-1$. The constant $\kappa_{0}^{2}$ is at this stage an arbitrary constant that can be redefined by a shift of the dilaton. This action represents a double perturbative expansion in both the string tension $\alpha^{\prime} \sim l_{s}^{2}$ and the string coupling $g_{s}^{2}=e^{2 \Phi}$. We should only trust this action for a large radius of curvature $\left(l_{s}^{2} \ll R_{c}\right)$ and weak coupling $\left(g_{s} \ll 1\right)$. In particular, if we wish to add string matter to the action we should respect these low energy and large radius approximations.

From the perspective of the worldsheet (CFT), the action (11) ensures that the worldsheet couplings $G_{M N}, B_{M N}$, and $\Phi$ are scale invariant by the vanishing of their renormal- 
ization group $\beta$ functions [12]. These couplings appear in the worldsheet action which is described by the nonlinear sigma model

$$
S_{0}=\frac{1}{\pi \alpha^{\prime}} \int d^{2} z\left[G_{M N}(X)+B_{M N}(X)\right] \partial X^{M} \bar{\partial} X^{N}+\alpha^{\prime} \mathcal{R}^{(2)} \Phi(X),
$$

where $\mathcal{R}^{(2)}$ is the worldsheet Ricci scalar, $X^{M}$ are the worldsheet fields, and $\partial(\bar{\partial})$ is the left (right) derivative on the $2 d$ worldsheet. One finds that by demanding the $\beta$ functions vanish for these couplings, we regain their usual equations of motion, where we intepret the couplings as the low energy supergravity fields in the target space. The CFT approach can be used to calculate S-matrix elements by expanding the couplings about their classical values,

$$
\begin{aligned}
G_{M N} & =G_{M N}^{(0)}+\tilde{h}_{M N}, \\
B_{M N} & =B_{M N}^{(0)}+\tilde{b}_{M N}, \\
\Phi & =\Phi^{(0)}+\tilde{\Phi},
\end{aligned}
$$

where the perturbations $\tilde{h}_{M N}, \tilde{b}_{M N}$, and $\tilde{\Phi}$ represent the specific string states of the graviton, antisymmetric tensor, and the dilaton, respectively. One can then include other string states by inserting additional vertex operators $\mathcal{O}(z, \bar{z})$ with (left, right) conformal dimension $(1,1)$ which represent marginal deformations of the CFT,

$$
S_{0} \longrightarrow S^{\prime}=S_{0}+\lambda \int d^{2} z \mathcal{O}(z, \bar{z})
$$

These marginal deformations take one CFT $S_{0}$ into another $S^{\prime}$, providing a connected set of CFTs that all represent the same physical theory [13]. This neighborhood around the CFT fixed point makes up the moduli space and the couplings of these marginal operators are the moduli. The motion of the moduli are then given by their RG flow, where $\lambda$ represents the infinitesimal motion. One problem of string theory is to determine a vacuum expectation value (VEV) for each field (or worldsheet coupling), thus fixing these moduli to some unique value. If we attempt to do this by including additional matter, we have argued that this should respect the low energy approximation. In the CFT, this 
means that the additional fields must correspond to exactly marginal deformations. So we see that by demanding conformal invariance and working in an effective theory, there are very tight constraints on the possibilities of adding additional matter. In particular, if we are to consider additional string states they should be nearly massless. We will see that such states arise after compactification and at special points in the moduli space.

Motivated by the Brandenberger-Vafa scenario [14], we now consider the decompactification of four dimensions resulting in $\mathcal{M}^{4} \times \mathcal{K}^{d}$ where $\mathcal{K}$ remains compactified and $\mathcal{M}^{4}$ corresponds to an Friedmann-Robertson-Walker (FRW) or time dependent cosmological background ${ }^{1}$. We assume that the four dimensions have expanded enough so that the curvature scale of $\mathcal{M}^{4}$ is much larger than the scale of the compact dimensions (i.e. $R_{c}^{(4)}>>R_{c}^{(d)}$ ). In this limit, the low energy degrees of freedom are given by the dimensionally reduced action,

$$
\begin{array}{r}
S=\frac{1}{2 \kappa_{4}^{2}} \int d^{4} x \sqrt{-g} e^{-2 \varphi}\left[R+4(\nabla \varphi)^{2}+\frac{1}{4} \nabla_{\mu} h^{a b} \nabla^{\mu} h_{a b}-\frac{1}{4} \mathcal{F}_{\mu \nu a} \mathcal{F}^{\mu \nu a}\right. \\
\left.-\frac{1}{12} H_{\mu \nu \lambda} H^{\mu \nu \lambda}-\frac{1}{4} H_{\mu \nu a} H^{\mu \nu a}-\frac{1}{4} H_{\mu a b} H^{\mu a b}-\frac{1}{12} H_{a b c} H^{a b c}\right],
\end{array}
$$

where $M, N=0 \ldots(D-1)$ refer to the full space-time, $\mu, \nu=0 \ldots 3$ denote the $\mathcal{M}^{4}$ directions, and $a, b, c=4 \ldots(D-1)$ denote the compact dimensions of $\mathcal{K}$. The $D$ dimensional metric is given by,

$$
G_{M N}=\left(\begin{array}{cc}
g_{\mu \nu}+h_{a b} A_{\mu}^{a} A_{\nu}^{b} & A_{\mu b} \\
A_{\nu a} & h_{a b}
\end{array}\right)
$$

and we have introduced the four dimensional dilaton $\varphi \equiv \Phi-\frac{1}{2} \ln \operatorname{det} h_{a b}$ and $2 \kappa_{4}^{2}=$ $16 \pi G=2 \pi \alpha^{\prime} g_{s}^{2}$. The compactification of $B_{M N}$ follows analogous to (6) with $B_{\mu \nu}^{D}=B_{\mu \nu}+$ $h_{a b} B_{\mu}^{a} B_{\nu}^{b}$. In general, we will be interested in internal metrics of the form $h_{a b}=\alpha^{\prime} e^{2 \sigma} \delta_{a b}$.

\footnotetext{
${ }^{1}$ More precisely, in 14 the geometry was taken as $\mathbb{R} \times T^{3} \times T^{6}$ where $T^{n}$ is the $n$ torus. At late times, when the $T^{3}$ has grown large the geometry is approximately $\mathcal{M}^{4} \times \mathcal{K}^{d}$ where $\mathcal{M}^{4}$ is Minkowski space-time.
} 


\section{A. String Spectrum on $M^{4} \times S^{1}$}

We now consider the case of one extra dimension with metric $G_{55}=\alpha^{\prime} e^{2 \sigma}$, where $\sigma$ is the radion and we approximate the large dimensions as Minkowski space-time $M^{4}$. We make this assumption for simplicity and note that our main results can be extended to more general cases, such as the $d=6$ compactification of the Heterotic string. The action (5) simplifies to

$$
S=\frac{1}{2 \kappa_{4}^{2}} \int d^{4} x \sqrt{-g} e^{-2 \varphi}\left(R+4(\partial \varphi)^{2}-(\partial \sigma)^{2}-\frac{1}{4} e^{2 \sigma} \mathcal{F}_{\mu \nu} \mathcal{F}^{\mu \nu}-\frac{1}{4} e^{-2 \sigma} B_{\mu \nu} B^{\mu \nu}-\frac{1}{12} H^{2}\right),
$$

where $H_{\mu \nu \lambda}=\partial_{[\mu} B_{\nu \lambda]}$ is the flux in four dimensions and $\mathcal{F}_{\mu \nu}$ contains the usual Kaluza-

Klein $(\mathrm{KK})$ vectors of mass $m^{2}=\frac{n^{2}}{R^{2}}$ resulting from the compactified metric i.e., $G_{\mu 5}$. In addition, we also get another $U(1)$ from the compactification of the higher dimensional antisymmetric two form $B_{\mu 5}$ resulting in the winding states with $m^{2}=\frac{\omega^{2} R^{2}}{\alpha^{\prime 2}}$.

We can also see these states arising from the more fundamental worldsheet action (2). Assuming the couplings don't depend on the extra dimension we find that the reduced action becomes

$$
\begin{aligned}
S_{4 p}= & \frac{1}{\pi \alpha^{\prime}} \int d^{2} z\left[G_{\mu \nu}(X)+B_{\mu \nu}(X)\right] \partial X^{\mu} \bar{\partial} X^{\nu}+\left[G_{\mu 5}(X)+B_{\mu 5}(X)\right] \bar{\partial} X^{\mu} \partial X^{5} \\
& +\left[G_{\mu 5}(X)-B_{\mu 5}(X)\right] \partial X^{\mu} \bar{\partial} X^{5}+G_{55}(X) \partial X^{5} \bar{\partial} X^{5}+\alpha^{\prime} \mathcal{R}^{(2)} \Phi(X),
\end{aligned}
$$

where $R \equiv G_{55}^{1 / 2}=\sqrt{\alpha^{\prime}} e^{\sigma}$ is the radius of the extra dimension. To manifest the chiral symmetry (right and left currents move independently on the string), we can define

$$
\begin{aligned}
& A_{\mu} \equiv A_{\mu}^{\text {Left }}=\frac{1}{2}\left(G_{\mu 5}+B_{\mu 5}\right), \\
& \bar{A}_{\mu} \equiv A_{\mu}^{\text {Right }}=\frac{1}{2}\left(G_{\mu 5}-B_{\mu 5}\right) .
\end{aligned}
$$

The mass of a string state is found by enforcing conformal symmetry mode by mode and results in the Virasoro constraints [12],

$$
\begin{array}{r}
M^{2}=\frac{n^{2}}{R^{2}}+\frac{\omega^{2} R^{2}}{\alpha^{\prime 2}}+\frac{2}{\alpha^{\prime}}(N+\bar{N}-2), \\
n \omega+N-\bar{N}=0,
\end{array}
$$


where the integers $n$ and $\omega$ label the momentum and winding charge and $N(\bar{N})$ correspond to the number of left (right) oscillators that are excited. We are interested in the massless states and we can see from (10) that for generic radii this means we are restricted to the states

$$
n=\omega=0 \quad N=\bar{N}=1
$$

The effective lagrangian representing the additional degrees of freedom from the string compactification takes form

$$
\mathcal{L}_{m}=(\partial \sigma)^{2}-\frac{1}{4 g^{2}}\left(F_{\mu \nu} F^{\mu \nu}\right)-\frac{1}{4 g^{2}}\left(\bar{F}_{\mu \nu} \bar{F}^{\mu \nu}\right),
$$

with

$$
F_{\mu \nu}=\partial_{[\mu} A_{\nu]} \quad \bar{F}_{\mu \nu}=\partial_{[\mu} \bar{A}_{\nu]}
$$

and the scalar is neutral and comes from $N=\bar{N}=1$ being taken in the compact direction. Thus, we have a $U_{L}(1) \times U_{R}(1)$ chiral gauge theory resulting from the compactification, along with the neutral scalar $\sigma$ giving the overall scale of the extra dimension. This doubling of the usual KK spectrum is an example of how strings lead to an enrichment of the usual symmetries. In summary, we have found that the low energy physics is described by dilaton gravity coupled to a $U_{L}(1) \times U_{R}(1)$ chiral gauge theory with a neutral scalar field.

As in the uncompactified case, we are interested in the fluctuations about the background values. For the new states resulting from compactification we have the lower dimensional analogs of (3) along with the new states

$$
\begin{aligned}
A_{\mu} & =A_{\mu}^{(0)}+A_{\mu}^{(3)}, \\
\bar{A}_{\mu} & =\bar{A}_{\mu}^{(0)}+\bar{A}_{\mu}^{(3)}, \\
G_{55} & =\alpha^{\prime}\left(1+\phi^{(33)}\right),
\end{aligned}
$$

where the first term on the right is the background value. Our notation for the left (right) going vector perturbation $A_{\mu}^{(3)}\left(\bar{A}_{\mu}^{(3)}\right)$ and scalar perturbation $\phi^{(33)}$ will become 
clear shortly. The vertex operators corresponding to the insertion of these states are given by

$$
\begin{aligned}
V^{(3)} & =A_{\mu}^{(3)} \partial X^{5} \bar{\partial} X^{\mu}, \\
\bar{V}^{(3)} & =\bar{A}_{\mu}^{(3)} \partial X^{\mu} \bar{\partial} X^{5}, \\
V^{(33)} & =\phi^{(33)} \partial X^{5} \bar{\partial} X^{5} .
\end{aligned}
$$

Note that these operator insertions correspond to the same states that appeared in the space-time matter lagrangian (12). We can identify $\phi^{(33)}$ with a perturbation of the compact radius away from the self dual radius and then $\phi^{(33)}=0$ corresponds to $R=\sqrt{\alpha^{\prime}}$. For $\sigma \ll 1$ we find $\sigma \sim \phi^{(33)} \sim \delta R$ gives the departure from the self dual radius.

Let us consider the simple string background $G_{\mu \nu}=\eta_{\mu \nu}, B_{\mu \nu}=0$, and $\Phi=\Phi_{0}$. As we have discussed, the low energy approximation requires the fluctuations in (15) to correspond to exactly marginal operators and be primary fields of dimension $(1,1)$. This requirement implies that they must obey the following equations [8],

$$
\square \phi^{(33)}=\square A_{\mu}^{(3)}=\square \bar{A}_{\mu}^{(3)}=0 \quad \partial^{\mu} A_{\mu}^{(3)}=\partial^{\mu} \bar{A}_{\mu}=0 .
$$

Once again we see that by demanding conformal invariance we obtain the expected equations of motion for the fields that would have followed from (12) with the covariant choice of the Lorentz gauge. We also see from these equations, it is clear that there is nothing to determine the VEV of $\phi^{(33)}$. This is a simple example of the inability of string theory to predict a unique vacuum of the theory. That is, each value of $\left\langle\phi^{(33)}\right\rangle$ corresponds to a different choice of vacuum for the same string theory. From the perspective of cosmology this implies that the radion $\phi^{(33)}$ is not stabilized in the low energy theory. This can be understood from (12), where we saw that with $n=\omega=0$, the radion $\phi^{(33)}$ is uncharged under the $U_{L}(1) \times U_{R}(1)$ and there is nothing to fix the scale. One might expect that including the winding and momentum modes could change this result, but we have seen that restricting to the low energy spectrum eliminates states that carry winding and mo- 
mentum charge. We will now see that this does not necessarily remain true if we consider regions of the moduli space near special radii, so-called ESPs.

\section{B. Enhanced Gauge Symmetry}

Upon examining the mass spectrum given by (10), we see that at the special radius $R=\sqrt{\alpha^{\prime}}$ there is a possibility of additional massless states.

These correspond to four new vectors,

$$
\begin{array}{lll}
N=0 \quad \bar{N}=1 \quad n=\omega= \pm 1 & V^{( \pm)}=A_{\mu}^{( \pm)} \bar{\partial} X^{\mu} \exp \left( \pm i \frac{2}{\sqrt{\alpha^{\prime}}} X^{5}\right) \\
N=1 \quad \bar{N}=0 \quad n=-\omega= \pm 1 & \bar{V}^{( \pm)}=\bar{A}_{\mu}^{( \pm)} \partial X^{\mu} \exp \left( \pm i \frac{2}{\sqrt{\alpha^{\prime}}} \bar{X}^{5}\right)
\end{array}
$$

four massless scalars, where $N$ or $\bar{N}$ are taken in the compact direction

$$
\begin{array}{lll}
N=0 \quad \bar{N}=1 \quad n=\omega= \pm 1 & V^{( \pm 3)}=\phi^{( \pm 3)} \bar{\partial} X^{5} \exp \left( \pm i \frac{2}{\sqrt{\alpha^{\prime}}} X^{5}\right) \\
N=1 \quad \bar{N}=0 \quad n=-\omega= \pm 1 & V^{(3 \pm)}=\phi^{(3 \pm)} \partial X^{5} \exp \left( \pm i \frac{2}{\sqrt{\alpha^{\prime}}} \bar{X}^{5}\right)
\end{array}
$$

and four massless scalars that are purely winding and purely momentum charged states

$$
\begin{array}{ll}
N=\bar{N}=\omega=0 \quad n= \pm 2 & V^{( \pm \pm)}=\phi^{( \pm \pm)} \exp \left( \pm i \frac{2}{\sqrt{\alpha^{\prime}}} X^{5}\right) \exp \left( \pm i \frac{2}{\sqrt{\alpha^{\prime}}} \bar{X}^{5}\right) \\
N=\bar{N}=n=0 \quad \omega= \pm 2 & V^{( \pm \mp)}=\phi^{( \pm \mp)} \exp \left( \pm i \frac{2}{\sqrt{\alpha^{\prime}}} X^{5}\right) \exp \left(\mp i \frac{2}{\sqrt{\alpha^{\prime}}} \bar{X}^{5}\right) .
\end{array}
$$

This concludes the massless spectrum at the $\operatorname{ESP} R=\sqrt{\alpha^{\prime}}$. We see that it is now consistent to include states with nontrivial winding and momentum into the low energy spectrum. These additional vectors (17) fill out the adjoint representation of $S U_{L}(2) \times$ $S U_{R}(2)$ enhancing the previous symmetry from $U_{L}(1) \times U_{R}(1)$. The eight scalars (18) and (19) combine with $\phi^{(33)}$ to transform as the $(\mathbf{3 , 3})$ adjoint representation of the gauge group. The T-duality that is usually associated with winding/momentum states can now be realized as a gauge rotation by $\pi$ in one of the $S U(2)$ 's which sends $\phi^{(33)}$ to $-\phi^{(33)}$ or $R$ to $\alpha^{\prime} / R$. Thus, T-duality arises from the action of a $\mathbb{Z}_{2}$ subgroup of the full 
$S U_{L}(2) \times S U_{R}(2)$ symmetry. This is an example of the fact that duality holds in the nonperturbative regime, since the gauge theory approach will remain valid there [13].

The requirement that these fields maintain conformal invariance, being primary operators of dimension $(1,1)$, is

$$
\square A^{(i)}=\square \bar{A}^{(i)}=\square \phi^{i j}=0 \quad \partial^{\nu} A_{\nu}^{i}=\partial^{\nu} \bar{A}_{\nu}^{i}=0,
$$

where $i, j=+,-, 3$. These are the expected equations resulting from (12) after lifting the theory to $S U_{L}(2) \times S U_{R}(2)$ and restricting to the self dual radius, i.e. $\left\langle\phi^{(33)}\right\rangle=0$. The field strength (13) is now given by the Yang-Mills theory

$$
\begin{aligned}
& F_{\mu \nu}^{i}=\partial_{\mu} A_{\nu}^{i}-\partial_{\nu} A_{\mu}^{i}+g \epsilon^{i j k} A_{\mu}^{j} A_{\nu}^{k}, \\
& \bar{F}_{\mu \nu}^{i}=\partial_{\mu} \bar{A}_{\nu}^{i}-\partial_{\nu} \bar{A}_{\mu}^{i}+g \epsilon^{i j k} \bar{A}_{\mu}^{j} \bar{A}_{\nu}^{k},
\end{aligned}
$$

and the scalars couple through the $(\mathbf{i}, \mathbf{0})$ and $(\mathbf{0}, \mathbf{j})$ gauge covariant derivatives

$$
\begin{aligned}
& \left(D_{\mu} \phi\right)^{a}=\partial_{\mu} \phi^{a}+g \epsilon^{a b c} A_{\mu}^{b} \phi^{c}, \\
& \left(\bar{D}_{\mu} \phi\right)^{a}=\partial_{\mu} \phi^{a}+g \epsilon^{a b c} \bar{A}_{\mu}^{b} \phi^{c},
\end{aligned}
$$

where the coupling $g$ is of $\mathcal{O}(1)$ for the states we are considering ${ }^{2}$.

\section{Higgs Mechanism in String Theory}

We have seen that at the $\operatorname{ESP}$ (i.e. $R=\sqrt{\alpha^{\prime}}$ ) the gauge symmetry is enhanced to $S U_{L}(2) \times S U_{R}(2)$. Let us consider the mass of the states (17), (18), and (19) away from $R=\sqrt{\alpha^{\prime}}$. We find

$$
M=\frac{\left|R^{2}-\alpha^{\prime}\right|}{R \alpha^{\prime}}=\frac{2}{\sqrt{\alpha^{\prime}}}|\sinh (\sigma)|,
$$

\footnotetext{
${ }^{2}$ For example, for the heterotic string the four dimensional gauge coupling is given by $g^{2}=4 \kappa^{2} / \alpha^{\prime}$, where $\kappa$ is the gravitational length and contains the dilaton expectation value. One can usually choose these values so that $g$ is order one, which is expected from the Yang-Mills theory. This implies that the string scale is close to the gravitation scale. For a complete discussion see 12 .
} 
where we have used $R=\sqrt{\alpha^{\prime}} e^{\sigma}$. Thus, we see that if the radion has a vanishing VEV these states remain massless, whereas if $\phi^{(33)} \neq 0$ the states become massive. This is an example of the Higgs Mechanism in string theory, where the role of the Higgs is played by the radion $\phi^{(33)}$. We can see the Higgs mechanism explicitly from (23) and (24). Consider the $(\mathbf{i}, \mathbf{0})$ covariant derivative for the scalar $\phi^{(+3)}$,

$$
\begin{aligned}
D_{\mu} \phi^{(+3)} & =\partial_{\mu} \phi^{(+3)}+g \epsilon^{+b c} A_{\mu}^{b} \phi^{c}, \\
& =\partial_{\mu} \phi^{(+3)}+g\left(A_{\mu}^{-} \phi^{(33)}-A_{\mu}^{3} \phi^{(-3)}\right) .
\end{aligned}
$$

As usual, we can perform a gauge rotation to fix the VEV of $\phi$ in the $(\mathbf{3}, \mathbf{3})$ direction $\left\langle\phi^{(33)}\right\rangle=\delta R$ and through the kinetic term this gives a mass to the vector $A_{\mu}^{(-)}$,

$$
\frac{1}{2}\left(D_{\mu} \phi^{(+3)}\right)^{2} \longrightarrow \frac{1}{2} g^{2}\left\langle\phi^{(33)}\right\rangle^{2}\left(A_{\mu}^{-}\right)^{2}=\frac{1}{2} g^{2} \delta R^{2}\left(A_{\mu}^{-}\right)^{2} .
$$

Through this and the other covariant derivative terms the four vectors $A^{ \pm}$and $\bar{A}_{\mu}^{ \pm}$get masses through their couplings to $\phi^{(33)}$, while the $A_{\mu}^{(3)}$ and $\bar{A}_{\mu}^{(3)}$ remain massless. Thus, we see that the $S U_{L}(2) \times S U_{R}(2)$ is broken to $U_{L}(1) \times U_{R}(1)$. As we have discussed, it is problematic in string theory to determine $\delta R^{2}$, since no potential exists for the scalars $\phi$. However, we argue in what follows that by considering the time dependence of the background, one is naturally led to a cosmological mechanism that breaks this degeneracy.

\section{AN EFFECTIVE POTENTIAL FOR RADION STABILIZATION}

We saw in the last section that the Higgs mechanism arises naturally in string theory near ESPs. In the simple $S^{1}$ compactification we considered, we found that the low energy degrees of freedom evolved in accordance with dilaton gravity coupled to a chiral gauge theory $U_{L}(1) \times U_{R}(1)$. This was true at generic radii and we found that there was no scale to fix the VEV of the radion. We also found that near the $\operatorname{ESP}$ (i.e. $R=\sqrt{\alpha^{\prime}}$ ) extra states (17), (18), and (19) became massless and we should include these states and their 
interactions in the effective theory ${ }^{3}$.

This suggests the following model for stabilizing the radion near the ESP. Initially the radion $\sigma$ begins at a generic point in the moduli space and the correct theory is that of dilaton gravity coupled to the $U_{L}(1) \times U_{R}(1)$ massless degrees of freedom. We assume the dilaton has been fixed ${ }^{4}$ and we consider the late time cosmology in an homogeneous and isotropic universe with metric,

$$
d s_{4}^{2}=-d t^{2}+e^{2 \lambda(t)} d \vec{x}^{2}
$$

where $\lambda(t)=\ln a(t)$ is the scale factor. The effective action for generic $\sigma$ is given by

$$
S_{e f f}=\int d^{4} x \sqrt{g}\left[R-\frac{1}{2}(\partial \sigma)^{2}-V_{e f f}\right]
$$

where $V_{\text {eff }}$ initially represents the contribution from the chiral $U(1)$ s, although near the self dual radius it should incorporate the effects due to the additional massless states.

Let us consider the background equations of motion first neglecting the backreaction near the ESP. The equations following from (29) are

$$
\begin{array}{r}
3 \dot{\lambda}^{2}=\frac{1}{2} \dot{\sigma}^{2}+\rho_{\text {sub }} \\
2 \ddot{\lambda}+3 \dot{\lambda}^{2}=-\frac{1}{2} \dot{\sigma}^{2}-p_{\text {sub }} \\
\ddot{\sigma}+3 \dot{\lambda} \dot{\sigma}=\frac{\partial V_{\text {eff }}}{\partial \sigma}
\end{array}
$$

where $\rho_{\text {sub }}$ and $p_{\text {sub }}$ represent the subdominant contribution from the $U_{L}(1) \times U_{R}(1)$ contained in $V_{\text {eff }}$ at generic radii. This contribution will be subdominant at early times, since the kinetic term has an equation of state $\rho=p$ and thus scales as $\rho=a^{-6}$. The

\footnotetext{
${ }^{3}$ Similar considerations were addressed from the supergravity perspective for the case of the heterotic string on $K 3 \times S^{1}$ and $K 3 \times T^{2}$ in 9 ] and [10], respectively.

${ }^{4}$ Since we are interested in late time cosmology, we will assume that the dilaton has already been stabilized. We anticipate this could be done either by fluxes, wrapped branes, or perhaps even by a variation of the mechanism we will describe.
} 
corresponding scale factor is $a(t) \sim t^{1 / 3}$ and $\dot{\lambda}=1 / 3 t$. In this limit we can ignore the potential in (32) and $\sigma$ is given for small $t$ as

$$
\sigma(t)=\sigma_{0}+v_{0} t
$$

We start the time evolution at $t=0$ when the field is closest to $R=\sqrt{\alpha^{\prime}}$, thus we see that $\sigma_{0}$ is a measure of how close the radion comes to the ESP. In [2], it was shown that by including the dilaton in the dynamics, along with the winding and momentum modes of the string, the radion will naturally pass through $\sigma=0$ and be localized around the $\mathrm{ESP}^{5}$. Motivated by this result we assume that $\sigma_{0}=0$, which is the most efficient case for particle production, since the states will be exactly massless there.

We proceed to address particle creation in a way analogous to the treatment in models of inflationary preheating [18], where here the role of the inflaton will be played by the radion. Since we are really discussing the creation of strings, one might wonder if we are justified in taking this approach. It was shown in [19] that string production can be considered in this way. There it was shown that it is enough to consider the production of strings, mode by mode, in the effective field theory approach. Using this approach we can think of each string mode as a scalar field with a time varying mass.

For example, let us consider the effects of producing one of the additional massless vectors that appears at the ESP. From the coupling in (27) we see that the additional states would lead to a potential

$$
V_{e f f}\left(\sigma, A_{\mu}\right)=\frac{1}{2}\left(\partial_{\mu} A_{\nu}\right)^{2}-\frac{1}{2} g^{2} \sigma^{2} A_{\mu} A^{\mu}
$$

where we have defined $A_{\mu} \equiv A_{\mu}^{(-)}$and we work in the Lorentz gauge $\partial^{\mu} A_{\mu}=0$. Note that we are neglecting the other Yang-Mills interactions, as these would lead to the same generic dynamics for $\sigma$. However, it would be interesting to include these interactions

\footnotetext{
${ }^{5}$ This result was for the full $10 D$ theory in the string frame. We note that this result came from trusting the low energy effective action while including the massive modes of the string. This seems to be an issue that requires further attention.
} 
in future work, as they are examples coming directly from string theory of the type of interactions recently considered in [20] as dark matter candidates.

From (34), we can identify $m(t)^{2}=g^{2} \sigma^{2}$ as a time dependent mass for $A_{\mu}$. As $\sigma$ approaches the ESP, the $A_{\mu}$ 's become massless and easy to create. Then, as $\sigma$ leaves the ESP these states will become massive resulting in backreaction and producing an attractive force pulling $\sigma$ back towards the ESP.

Let us consider the time dependent frequency of a particular Fourier mode $A_{k}^{\mu}$

$$
\omega_{k}(t)=\sqrt{\vec{k}^{2}+g^{2} \sigma^{2}(t)}
$$

A particular mode becomes excited when the non-abiabaticity parameter satisfies $\dot{\omega} / \omega^{2} \geq$ 1. When this condition holds for a particular mode, it results in particle production and an occupation number

$$
n_{k}=\exp \left(-\frac{\pi \vec{k}^{2}+g^{2} \sigma_{0}^{2}}{g v_{0}}\right) .
$$

Recall that we can take $\sigma_{0}=0$ and $g$ in our case is a positive constant of order unity coming from the coupling of the winding and momentum modes. The energy density of produced particles is given by

$$
\rho_{A}=\int \frac{d^{3} k}{(2 \pi)^{3}} n_{k} \omega_{k} \approx g|\sigma(t)| N,
$$

with $N \sim\left(g v_{0}\right)^{3 / 2}$. Thus, comparing this to (301) we see that the initial kinetic energy associated with the radion $\frac{1}{2} v_{0}^{2}$ is dumped into production of $A_{\mu}$ particles as the radion passes through the ESP. Given a large enough $v_{0}$, the radion will continue its trajectory and the modes will become massive as we have seen. This results in an always attractive force of magnitude $g N$ pointing the radion back towards the ESP. The effective equation for $\sigma$ including the backreaction is then given by

$$
\ddot{\sigma}+3 \dot{\lambda} \dot{\sigma}=-g N(t)
$$

This process will continue with each pass of the radion, until all of its initial kinetic energy has been used up and it settles to the self dual radius. Therefore, we are led to 
the conclusion that the additional states associated with the enhanced symmetry result in a fixed value for the radion at the self dual radius.

One immediate concern might be whether this method is stable to perturbations. Moreover, one could worry that the initial kinetic energy of the radion is so high that the force associated with the backreaction is not enough to over come its inertia. Both of these problems are overcome by considering the Hubble friction associated with the second term in (38). One expects this friction to damp out any perturbations and should actually enhance the stabilization mechanism. This was discussed in models of string gas cosmology [4] and a similar conclusion was reached in 7]. Moreover, it was shown in 4] that once we switch to the effective theory the Hubble friction is enough to keep the radion evolving slowly compared to the growth of the three large dimensions. We conclude that Hubble friction combined with the ESP backreaction should be more than adequate to stabilize the radion at the self dual radius.

\section{CONCLUSIONS}

We have found that by considering the string Higgs effect it is possible to generate a potential for the radion resulting from the backreaction of the additional low energy states that emerge at the ESP. These low energy states carry both winding and momentum charge, justifying their inclusion in low energy effective actions where the dimensions are taken initially to be near the self dual radius. This includes models of Brane Gas Cosmology, which address the issue of stabilization of extra dimensions from the higher

dimensional perspective [2, 3]. This complements our approach here, where we have demonstrated the stabilization from the lower dimensional perspective without the help of the dilaton.

When considering the inclusion of these additional modes we have neglected much of the richness of the nonabelian gauge theory. That is, we focused on the coupling of the vectors and radion arising from the covariant derivatives in (12), but we have neglected 
the interactions and self interactions of the fields. Including these additional interactions would be interesting for a number of reasons. They offer a concrete way to include the interactions of winding and momentum modes into models of Brane Gas Cosmology. Including additional interactions would also offer an example, coming directly from string theory, of the dark matter candidates recently proposed in [20].

Finally, it would be interesting to see if this mechanism can be combined with models of flux compactifications. As we mentioned in the introduction, these compactifications can fix all the moduli in principle, however the fixing of the overall scale of the extra dimensions seems incompatible with inflation [6]. Since this is precisely the radion, in principle, the two methods could be combined to fix all the moduli. One problem that prevents this from immediately being realized is that in these models one usually works in Type IIB theory and the compactification manifold is taken to be of the Calabi-Yau type. These manifolds are chosen because they offer the most realistic particle spectrum for Type IIB theories. In addition, they possess $S U(3)$ holonomy, which implies that the low energy string spectrum does not admit one cycles. As a result there is not an ESP corresponding to the self-dual radius and the methods we have suggested in this paper need not apply. However, our methods do readily apply to toriodal compactifications of the Heterotic string and work is currently underway to explore the additional role of fluxes. It will be interesting to see if slow-roll brane inflation can be realized in such a picture.

In closing, we hope the reader has been left with the idea that enhanced symmetry combined with cosmological evolution presents a viable mechanism for fixing moduli, but is in need of further study.

\section{Acknowledgments}

I would especially like to thank Steve Gubser for the initial motivation for undertaking this project. I would also like to thank Antal Jevicki, Daniel Kabat, David Lowe, 
Liam McAllister, and Cumrun Vafa for useful discussions, and Thorsten Battfeld, Robert Brandenberger and Sera Cremonini for comments on the manuscript. Financial support was provided by NASA GSRP.

[1] For foundational ideas see:

R. H. Brandenberger and C. Vafa, "Superstrings In The Early Universe," Nucl. Phys. B 316, 391 (1989);

A. A. Tseytlin and C. Vafa, "Elements of string cosmology," Nucl. Phys. B 372, 443 (1992) arXiv:hep-th/9109048;

S. Alexander, R. H. Brandenberger and D. Easson, "Brane gases in the early Universe," Phys. Rev. D 62, 103509 (2000) arXiv:hep-th/0005212;

R. Easther, B. R. Greene, M. G. Jackson and D. Kabat, "Brane gas cosmology in M-theory: Late time behavior," Phys. Rev. D 67, 123501 (2003) arXiv:hep-th/0211124.

For later generalizations see [4] and references within.

[2] S. Watson and R. Brandenberger, "Stabilization of extra dimensions at tree level," JCAP 0311, 008 (2003) arXiv:hep-th/0307044.

[3] S. P. Patil and R. Brandenberger, "Radion stabilization by stringy effects in general relativity and dilaton gravity," arXiv:hep-th/0401037;

B. A. Bassett, M. Borunda, M. Serone and S. Tsujikawa, "Aspects of string-gas cosmology at finite temperature," Phys. Rev. D 67, 123506 (2003) arXiv:hep-th/0301180.

[4] T. Battefeld and S. Watson, "Effective field theory approach to string gas cosmology," arXiv:hep-th/0403075.

[5] S. B. Giddings, S. Kachru and J. Polchinski, "Hierarchies from fluxes in string compactifications," Phys. Rev. D 66, 106006 (2002) arXiv:hep-th/0105097.

[6] S. Kachru, R. Kallosh, A. Linde, J. Maldacena, L. McAllister and S. P. Trivedi, "Towards inflation in string theory," JCAP 0310, 013 (2003) arXiv:hep-th/0308055. 
[7] L. Kofman, A. Linde, X. Liu, A. Maloney, L. McAllister and E. Silverstein, "Beauty is attractive: Moduli trapping at enhanced symmetry points," arXiv:hep-th/0403001.

[8] J. Bagger and I. Giannakis, "Higgs mechanism in string theory," Phys. Rev. D 56, 2317 (1997) arXiv:hep-th/9703202.

[9] T. Mohaupt and M. Zagermann, "Gauged supergravity and singular Calabi-Yau manifolds," JHEP 0112, 026 (2001) arXiv:hep-th/0109055.

[10] J. Louis, T. Mohaupt and M. Zagermann, "Effective actions near singularities," JHEP 0302, 053 (2003) arXiv:hep-th/0301125.

[11] J. E. Lidsey, D. Wands and E. J. Copeland, "Superstring cosmology," Phys. Rept. 337, 343 (2000) arXiv:hep-th/9909061.

[12] J. Polchinski, "String Theory. Vol. 1: An Introduction To The Bosonic String," "String Theory. Vol. 2: Superstring Theory And Beyond".

[13] A. Giveon, M. Porrati and E. Rabinovici, "Target space duality in string theory," Phys. Rept. 244, 77 (1994) arXiv:hep-th/9401139.

[14] R. H. Brandenberger and C. Vafa, "Superstrings In The Early Universe," Nucl. Phys. B 316, 391 (1989).

[15] S. S. Gubser, "String production at the level of effective field theory," arXiv:hep-th/0305099

[16] A. E. Lawrence and E. J. Martinec, "String field theory in curved spacetime and the resolution of spacelike singularities," Class. Quant. Grav. 13, 63 (1996) arXiv:hep-th/9509149.

[17] S. Watson and R. Brandenberger, "Linear perturbations in brane gas cosmology," JHEP 0403, 045 (2004) arXiv:hep-th/0312097;

S. Watson, "UV perturbations in brane gas cosmology," arXiv:hep-th/0402015.

[18] J. H. Traschen and R. H. Brandenberger, "Particle Production During Out-Of-Equilibrium Phase Transitions," Phys. Rev. D 42, 2491 (1990);

L. Kofman, A. D. Linde and A. A. Starobinsky, "Towards the theory of reheating after inflation," Phys. Rev. D 56, 3258 (1997) arXiv:hep-ph/9704452.

[19] A. E. Lawrence and E. J. Martinec, "String field theory in curved spacetime and the resolu- 
tion of spacelike singularities," Class. Quant. Grav. 13, 63 (1996) arXiv:hep-th/9509149;

S. S. Gubser, "String production at the level of effective field theory," arXiv:hep-th/0305099.

J. J. Friess, S. S. Gubser and I. Mitra, "String creation in cosmologies with a varying dilaton," arXiv:hep-th/0402156.

[20] S. S. Gubser and P. J. E. Peebles, "Structure formation in a string-inspired modification of the cold dark matter model," arXiv:hep-th/0402225. 